\title{
Advanced Launcher design options for Electron Cyclotron Current Drive on ITER based on Remote Steering
}

\author{
M.F. Graswinckel ${ }^{1}$, W.A. Bongers ${ }^{1}$, M.R. de Baar ${ }^{1}$, M.A. van den Berg', \\ G. Denisov ${ }^{4}$, A.J.H Donné ${ }^{1}$, B.S.Q. Elzendoorn ${ }^{1}$, A.P.H. Goede ${ }^{1}$, R. Heidinger ${ }^{5}$, \\ S. Kuzikov ${ }^{4}$, O.G. Kruijt ${ }^{1}$, B. Kruizinga ${ }^{2}$, A. Moro ${ }^{3}$, E. Poli ${ }^{6}$, D.M.S. Ronden ${ }^{1}$, \\ G. Saibene ${ }^{7}$, D.J. Thoen ${ }^{1}$ and A.G.A. Verhoeven ${ }^{1}$ \\ ${ }^{1}$ FOM-Institute for Plasma Physics Rijnhuizen, Association EURATOM-FOM, \\ 3430 BE Nieuwegein, The Netherlands, http://www.rijnhuizen.nl/ \\ ${ }^{2} \mathrm{TNO}$, Delft, The Netherlands \\ ${ }^{3}$ Istituto di Fisica del Plasma, CNR, Ass. EURATOM-ENEA-CNR, Milano, Italy \\ ${ }^{4}$ IAP, Nizhny Novgorod, Russia, \\ ${ }^{5}$ FZK, Karlsruhe, Euratom Association, Germany \\ ${ }^{6}$ IPP, Garching, Euratom Association, Germany \\ ${ }^{7}$ EFDA Close Support Unit, Garching, Germany
}

Email main authors: W.A.Bongers@Rijnhuizen.nl, M.F.Graswinckel@Rijnhuizen.nl, Emanuele.Poli@ipp.mpg.de, moro@ipf.cnr.it

\begin{abstract}
Electron Cyclotron Current Drive (ECCD) will become the main scheme on ITER for the stabilization of neoclassical tearing modes (NTM) and the control of sawtooth oscillations. The effectiveness of this scheme forms the basis for the requirements of the ITER Upper Port Launcher (UPL). These requirements include the need for steering the mm-wave beam to the pertinent magnetic flux surfaces where the instability develops. Several concepts have been explored; Front Steering where a system of movable mirrors is situated at the plasma-facing end of the launcher; Remote Steering (RS) where the movable mirror system is situated at the back end of the launcher system. The advantage of the Remote Steering concept is that moving parts are placed away from the plasma in a secondary vacuum system, less exposed to plasma neutron flux, thus offering advantages in reliability and safety of the machine. Also, contamination of the beam steering unit by $\mathrm{Be}$ and $\mathrm{T}$ is less of a problem. However, beam-focusing properties of the RS launcher deteriorate with increased steering angle, which limits the NTM stabilisation performance as compared with Front Steering. In this paper, recent improvements in the RS design are presented. The stabilisation efficiency calculated reaches values that meet most of the ITER requirements. Improvements include non-spherical front mirrors and a tapered Square Corrugated Waveguide. Rather than steering all mm-wave beams over the entire vertical height range specified, RS launchers with a limited steering range are explored, yielding a performance improvement of $25 \%$ with an acceptable mm-wave heat load of $5.7 \mathrm{MW} / \mathrm{m}^{2}$ on the front mirrors. This result leads to a dual launcher concept with one set of beams dedicated to control NTMs at the outward lying $q=2$ surface and another set of beams dedicated to control NTMs at the inward lying $q=3 / 2$ surface in combination with saw teeth control at the innermost $q=1$ surface.
\end{abstract}

PACS numbers: $52.55 . \mathrm{Fa}, 84.40 . \mathrm{Ba}$ 


\section{Introduction}

The main objective of the ITER Upper Port Launcher (UPL) is to generate Electron Cyclotron Current Drive (ECCD) for the stabilization of neo-classical tearing modes (NTMs) that may develop at the $q=2 / 1$ and $q=3 / 2$ magnetic flux surfaces of the ITER plasma. In addition, the UPL is required to control the amplitude and frequency of sawtooth oscillations at the $q=1$ surface in support of the Equatorial Launchers (EL) dedicated to this task.

NTMs are triggered by small disturbances, locally reducing the pressure gradient at rational q surfaces, which gradient drives the bootstrap current $j_{b s}$. The consequent reduction in current density causes magnetic islands, i.e. tearing modes, to grow. NTMs degenerate global confinement and will prevent the ITER-plasma from reaching the pressures needed for substantial fusion power generation. Stabilization of NTMs can be achieved by restoring the current density profile through EC-wave driven current density $j_{c d}$.

Two alternative designs of the ITER UPL have been proposed: Front Steering (FS) with rotating mirrors situated near the plasma [1] and Remote Steering (RS) with rotating mirrors situated in the secondary vacuum of the launcher, about 5 meters away from the plasma [2]. The RS concept is based on the Talbot effect [3], first suggested for use in the UPL by [4], and analysed in detail by [5]. Individual beams are coupled into a Square Corrugated Waveguide (SCW) with dimensions such as to reproduce the input phase and amplitude of the beam at the output of the waveguide. By steering the input beam, the output beam follows this steering.

The main advantage of the FS concept is the wide range of steering achieved in combination with good beam focussing properties, allowing superior access to a wide range of plasma surfaces, whilst heat-loads on the movable mirrors are limited. The main advantage of the RS concept is a steering mechanism shielded from direct plasma exposure, with neutron bombardment on water-cooled moving parts reduced, yielding inherent advantages in safety and reliability of the machine. However, the down side is a restricted beam steering range compared with FS, a lower efficiency in driving current due to a wider beam deposition profile and a substantial heat-load on the front mirrors. In this paper, advances in performance through optimisation of the RS concept are presented. Preliminary results are published in [6].

In order to assess the RS design for NTM and sawtooth control capability, the EC current drive performance has been calculated for three ITER reference scenarios. These are the fully inductive scenario $2\left(I_{p}=15 \mathrm{MA}, B_{t}=5.3 \mathrm{~T}\right)$, the hybrid scenario $3 \mathrm{a}\left(I_{p}=12 \mathrm{MA}, B_{t}=5.3 \mathrm{~T}\right)$ and the low q scenario $5\left(I_{p}=17 \mathrm{MA}, B_{t}=5.3 \mathrm{~T}\right)$. In order to reach the $q=2 / 1$ and $q=3 / 2$ surfaces in these scenarios, the beam steering range must access the poloidal flux surfaces over the range $0.64<\rho_{p}<0.93$, where $\rho_{p}$ is the normalised plasma radius defined as the square root of the poloidal flux. Including access to the $q=1$ surface, the beam steering range needs to be extended to cover the range $0.35<\rho_{p}<0.93$ [7].

The ratio $\eta_{n t m}$ between the electron cyclotron wave driven current density $j_{c d}$ and the local bootstrap current density $j_{b s}$ expresses the efficiency by which the launcher is capable to stabilize NTMs [8]. For full NTM stabilization, $\eta_{n t m}$ should exceed 1.2 on the NTM prone surfaces [9]. A criterion to control the sawteeth is based on control of the magnetic shear $\mathrm{r} / q \mathrm{~d} q / \mathrm{d} r$ at the $q=1$ surface, again through electron cyclotron wave driven current [10].

The mm-wave power used for the calculation of $\eta_{n t m}$ is $20 \mathrm{MW}$ injected into the ITER plasma. This power is generated by 24 gyrotrons of $1 \mathrm{MW}$ power each at an estimated transmission loss of $4 \mathrm{MW}$. An installed power upgrade to $48 \mathrm{MW}$, possibly achieved by $2 \mathrm{MW}$ rated gyrotrons, is presently under consideration. The power loading calculations presented in this paper are all based on $2 \mathrm{MW}$ of power generated per gyrotron, anticipating on the power upgrade.

This paper describes recent advances in performance enhancement of the RS launcher concept. It assesses the Remote Steering performance of the ITER Upper Port Launcher in terms of current drive 
efficiency and shows for which ITER scenarios NTM stabilization requirements can be met. In section 2 , the basic design of the remote steered launcher is summarised. In section 3, the method for performance analysis and optimization is explained. In section 4, the performance results are presented. Section 5 discusses the validity of the computations and looks at possible future developments. Section 6 summarises and concludes.

\section{Design of the ITER Upper Port Launcher based on Remote Steering}

\subsection{General layout}

The Remote Steering design of the ECCD Upper Port Launching system on ITER comprises 24 beam lines divided over 4 Upper Ports transporting mm wave beams of frequency $170 \mathrm{GHz}$ rated at 1 to 2 MW of power each [2]. The steering system projects the mm-wave beam onto a Square Corrugated Waveguide (SCW) of length such that the input beam profile is imaged at the output, producing a steered mm-wave output beam [5]. The steering mechanism is placed in the secondary vacuum system of ITER where the neutron flux is reduced. A diamond vacuum window situated at the entrance of the SCW separates the steering system from the primary vacuum of ITER. It blocks Tritium and Beryllium contamination under normal operation conditions. The diamond window is coupled to a vacuum valve, which can be used to isolate the system from the ITER vacuum in case of window failure. At the front end of the launcher, a set of fixed mirrors is mounted in a periscope or dog-leg configuration inside the blanket section. This mirror configuration is designed to limit the neutron flux inside the launcher. The basic layout of the ITER ECCD Upper Port Plug is shown in Figure 1.

The UPL needs to fit within the spatial envelope of the ITER Upper Port Plug. The internal crosssection of the port plug is of near trapezoidal shape, $820 \mathrm{~mm}$ high, and 640 (420) $\mathrm{mm}$ wide at the base (top) respectively with an end to end length of approximately $6 \mathrm{~m}$. Radiation shielding is required to reduce the neutron fluence to less than $10^{20} \mathrm{n} \mathrm{m}^{-2}$ at the vacuum window over the ITER operational lifetime. To this end water-cooled stainless steel shielding blocks surround the waveguides. Neutronics calculations have shown that the fast neutron fluence at the diamond window is well below the design specification of $10^{20} \mathrm{n} \mathrm{m}^{-2}$ accumulated over the ITER operational life time [11]. The mechanical design should allow for maintenance and repair. This includes remote handling of the trapezoidal structure of the port plug taking due account of the leeway available in the torus hall. The cooling water temperature and its chemical composition are specific to ITER. Details on how the mechanical requirements are met are given in [12].

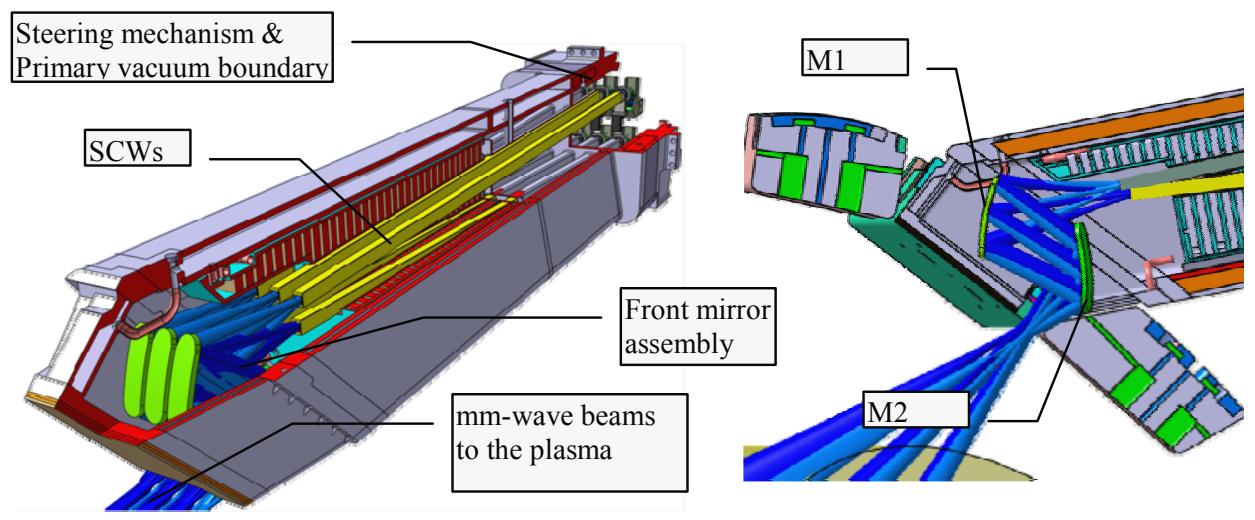

Figure 1 Left. Mechanical layout of the Upper Port Launcher based on Remote Steering showing the Square Corrugated Waveguides (SCW) integrated in the ITER port plug. The waveguides are arranged in two rows of three, i.e. an upper and lower row. The steering mechanism at the back end of the SCW is separated form the ITER vacuum system by a diamond vacuum window and a valve. 
Figure 1 Right, shows the fixed front mirror assembly integrated with the ITER blanket section.

\subsection{Thermo-Mechanical constraints}

The design of the UPL is subject to thermo-mechanical constraints, determined mainly by the front mirror system. The material selected for the mirror surfaces is $\mathrm{CuCrZr-IG}$, which combines excellent reflection properties for $170 \mathrm{GHz}$ waves with good thermal and mechanical properties, allowing an upper limit to the peak power load of $10 \mathrm{MW} / \mathrm{m}^{2}$. The maximum mirror surface temperature of the $\mathrm{CuCrZr}$-IG alloy is $240^{\circ} \mathrm{C}$, set by the limit of elastic deformation (negligible creep temperature).

The power loading calculation takes into account the mm-wave loss at the reflecting surface at $240^{\circ} \mathrm{C}$ surface temperature and includes a safety factor of 1.3 for the non-plasma facing mirrors and a factor 2 for plasma facing mirrors. This safety factor allows for degradation in the reflection properties of the mirrors, for example by material deposition and erosion. The change in beam optics due to thermal deformation of the mirrors during the beam pulse has been shown to stay within acceptable limits $(<10 \%$ change in focal length).

Heat loads on the mirror system calculated refer to mm-wave beam loads only. The radiation and neutron flux from the plasma cause an additional heat load. However, this additional heat load is calculated to be less than $10 \%$ of the mm-wave heat load and can therefore be ignored for the present analysis [11]. Peak power densities are evaluated for $2 \mathrm{MW}$ of power transmission per waveguide, consistent with an upgrade in total injected mm-wave power to $40 \mathrm{MW}$.

The rotating mirror system of the remote steering unit at the rear end of the $\mathrm{SCW}$ is designed to undergo $8.5 \times 10^{5}$ cycles [13] during the ITER operational lifetime, which comprises $3 \times 10^{4}$ ITER discharge pulses of $500 \mathrm{~s}$ duration each. This refers to the mirror movement mechanism subject to neutron bombardment. Fatigue of the mirror surfaces due to thermal cycling, induced by beam steering and beam modulation, is limited by staying below the $240{ }^{\circ} \mathrm{C}$ negligible creep temperature. Both fixed front and rotating rear mirror are calculated to be able to exceed $6 \times 10^{4}$ full heat cycles assuming that thermal effects of power modulation at NTM frequencies $(\sim 1 \mathrm{kHz})$ and beam movement over the mirror surface during steering can be neglected [14]. Compliance with ITER requirements has to be confirmed by a complete thermo-mechanical finite element analysis of the mirrors including fatigue over $8.5 \times 10^{5}$ beam steering cycles.

\section{Methods of launcher performance analysis and optimisation}

The method of launcher optimization consists of a non-linearly constrained maximization of the quasioptical parameters in terms of averaged scaled $\eta_{n t m}$ values. Here, the poloidal (vertical) beam dimensions at the location of wave absorption serve as a proxy to $\eta_{n t m}$ [15]. Spatial envelope and heat load on the individual beam line components serve as a constraint. After optimization of the optical system the model is re-iterated with the mechanical design model CATIA for mechanical inconsistencies and the design is adjusted where necessary. This cycle is repeated until a consistent design is achieved. The optimisation is furthermore constrained by $\eta_{n t m}$ values that are sub-marginal for some scenarios at the expense of $\eta_{n t m}$ values that are well above threshold at other scenarios. The design is then validated by carrying out a full calculation of $\eta_{n t m}$ values, including plasma transport, wave absorption and current drive of the $\mathrm{mm}$ waves injected by the optimised launcher. The result may lead to further iteration.

\subsection{Optimization of the optics}

The launcher model is divided into three subsystems; the steering mechanism, the SCW and the front mirror system. The optimization algorithm is based on a constrained 19 dimensional non-linear maximization which uses simplex, generalized-reduced-gradient, and branch-and-bound methods to find an optimal solution and sensitivity information. Constraints are defined on the 19 input 
parameters such as mirror position and curvature and SCW position and orientation, as well as on 16 derived output quantities such as heat-loads, the maximum beam size that can be handled by the mirrors, the beam truncation losses at the SCW entrance aperture and the minimum waist size of the beams entering the SCW. The most sensitive parameters are found to be: front mirror in-plane curvatures and steering mirror curvatures and positions.

Quasi-optical computations are based on Gaussian beam propagation [16]. The imaging properties of the corrugated square waveguide are derived from a full electro-magnetic calculation of the wave propagation inside the waveguide [17]. The SCW transforms the Gaussian input beam to an output beam with the same waist size but with a position slightly shifted in lateral direction, depending on the steering angle. For a steering angle $\gamma=+/-10.73^{\circ}$ the input beam waist is exactly imaged in size and position. In contrast to the FS concept, the beam focusing properties of the RS concept are coupled to the output steering range. A wider steering range goes at the expense of the beam quality. In the present design, the output steering angle $\gamma$ is limited between $-12^{\circ}$ and $+12^{\circ}$ with respect to the waveguide axis.

Three ways to reduce the heat load on the front mirrors have been explored; enlarging the distance from the SCW to the first front mirror, reducing the waist size at the input of the SCW and omitting a part of the sidewalls of the SCW.

- Enlarging the distance from the SCW to the front mirrors is no option due to limitations on the size of the front mirror that needs to fit within the spatial envelope of the port plug blanket section.

- A smaller beam waist at the entrance of the SCW results in a smaller beam waist at the exit of the SCW. This goes with a larger beam divergence towards the front mirrors, lowering heatloads and allowing better focusing. The problem is that a small entrance waist causes higher order modes to propagate inside the SCW resulting in higher losses and thus reducing the steering range of the SCW.

- The third option, removing a part of the SCW sidewalls parallel to the steering plane [18], allows for a larger beam divergence perpendicular to the steering plane, good for heat load reduction at the front mirror, whilst preserving a small beam divergence in the poloidal (vertical) plane required for efficient current drive. This option favourably decouples the optomechanical constraints into two independent optical planes. The scheme could be refined by mechanically linking the horizontal sidewalls of neighbouring SCWs producing a compact modular design of the top and bottom rows of waveguides. The consequences of this modification to the RS design are currently under investigation.

\subsection{General Astigmatism}

General astigmatism shows up when an astigmatic wave front is reflected by a curved surface with its planes of symmetry oriented arbitrarily with respect to the incoming beam. It is an intrinsic feature of the front mirror dog-leg configuration employed in the RS design, where two double curvature front mirrors M1 and M2 (Figure 1) are non symmetrically arranged with respect to the SCW plane of symmetry [19].

Including general astigmatism in the quasi-optical calculation produces a rotation of the beam spot ellipse, which needs to be taken into account when providing the initial conditions for beam tracing through the plasma. Beam tracing through the plasma has been carried out by means of the TORBEAM code [20], which includes general astigmatism and diffraction effects [21] and [22] neglected by the conventional ray tracing method. Results presented in Section 4.1 show that general astigmatic effects are of modest magnitude only, as comparison with previous results demonstrates [15].

As noted above, the double curvature front mirror M1 has its principal planes of symmetry, defined by the two mirror curvatures, oriented at an angle to the plane of symmetry of the incident simple astigmatic beam, defined by the SCW coordinate frame. This generates general astigmatism after 
reflection at the M1 mirror, which needs to be included in the calculation of reflection on the M2 mirror. Previously [19] [15], beam characteristics were calculated in simple astigmatic approximation, assuming constant curvatures along the principal directions of the double curvature mirror and independent propagation in the two astigmatism directions. Advanced RS launcher designs featuring elliptical mirrors require general astigmatic treatment, in order to take full account of the focal properties varying over the reflecting surfaces as a function of the steering angle $\gamma$ [24].

The input parameters for the TORBEAM code are obtained using the following method. The input steering mechanism is fed by a horn antenna, which is connected to the corrugated circular waveguide transmission line fed by the gyrotrons. The horn antenna improves coupling to the fundamental mode Gaussian beam so that for the remaining beam propagation calculations, Gaussian beam optics can be employed. For each steering angle of the steering system the input beam is transformed and produces a waist characterized by size and location that is radiated into the SCW. The SCW re-radiates this beam with the same parameters at mirrored angle, as was experimentally verified [23]. Starting from these parameters, beam optics calculations were performed [24] to characterize the resulting beams in terms of beam widths, curvature radii of wave fronts and orientation, for both spot ellipse and phase ellipse, representing the curves of constant intensity and constant phase respectively.

Beyond mirror M1, the complex formalism for general astigmatic beams is introduced [25]. The complex rotation angle $\varphi$, one of the key parameters for treatment of general astigmatism, is calculated using the initial conditions of the beams as a function of the steering angle $\gamma$. This allows determining the orientation angles $\varphi_{W}$ and $\varphi_{R}$ of the spot ellipse and of the phase front curvature respectively in the fixed coordinate system of the beam. These angles correspond to the orientations of the two rotating coordinate systems $\left(\xi_{w}, \eta_{w}\right)$ and $\left(\xi_{R}, \eta_{R}\right)$ of the beam in which the complex amplitude assumes the form

$$
\Psi(x, y, z)=A(z) \exp \left[-\left(\frac{\xi_{W}{ }^{2}}{w_{\xi}^{2}}+\frac{\eta_{W}{ }^{2}}{w_{\eta}^{2}}\right)\right] \cdot \exp \left[-j \frac{k}{2}\left(\frac{\xi_{R}{ }^{2}}{R_{\xi}}+\frac{\eta_{R}{ }^{2}}{R_{\eta}}\right)\right]
$$

where $w_{\xi, \eta}$ and $R_{\xi, \eta}$ are the principal beam widths and curvature radii respectively.

Figure 2 left shows the principal beam widths $w_{\eta}$ (upper set of curves) and $w_{\xi}$ (lower set of curves) for the short limited range launcher option (section 4.1), both for the upper row of beams (UR red curves) and lower row of beams (LR blue curves) as the beam propagates in vacuum at zero degree steering angle $\gamma$. The beams are found to be well elongated, with a major/minor beam width ratio $w_{\eta} / w_{\xi}$, in the absorption regions ranging from 1.48 to 2.72 over the range of steering angles. An arrow indicates where plasma absorption is expected to take place. As seen in Figure 2 right, the beam spot orientation varies with propagation distance in vacuum, rotating from upright to flat shape at the resonance region, showing general astigmatic features to emerge.
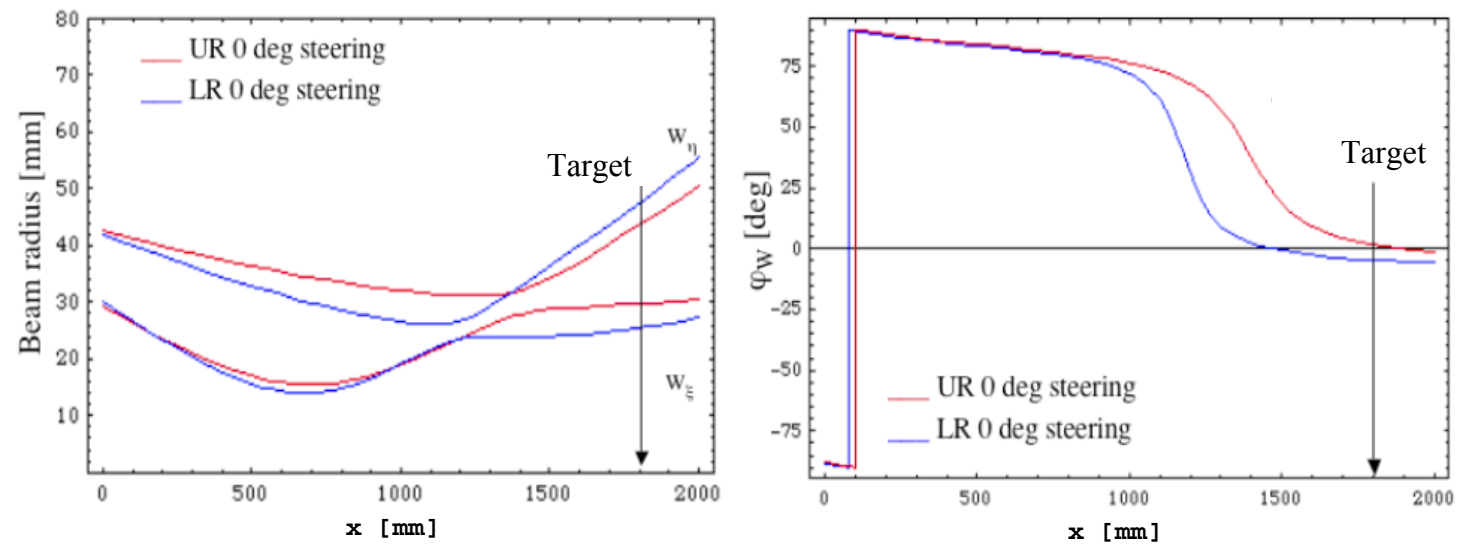
Figure 2. General astigmatic mm-wave beam propagation in vacuum. Left: Principal beam radii for the minor beam width $w_{\xi}$ (lower set of curves) and major beam width $w_{\eta}$ (upper set of curves) for the upper row of beams (UR red curve) and lower row of beams (LR blue curve) as a function of the distance $\mathrm{x}$ measured from mirror M2. The arrow indicates the location of plasma wave absorption. Right: The beam spot orientation $\varphi_{W}$ of the minor beam size $w_{\xi}$, is plotted as a function of distance $\mathrm{x}$ along the beam axis. The case of 0 degree beam steering for both upper row of beams (red curves) and lower row of beams (blue curves) is shown. During propagation the beam is shown to flip from upright to flat shape.

In Figure 3 the cross section of the beam spot at the position of mirror M2 (dashed line) and at the position of plasma absorption (solid line) is shown in cross sectional view for the range of beam steering angles $\gamma$. The orientation varies with propagation distance in vacuum, yielding a beam spot ellipse after mirror M2 with minor beam width $w_{\xi}$, in near vertical direction rotated at the resonance region to near horizontal direction (upper row of beams). This result is unfavourable in terms of $\eta_{n t m}$ performance, which requires a small beam width in vertical (poloidal) direction. The beam spot sizes $w_{\xi, \eta}$, the wave front curvatures $R_{\xi, \eta}$ and the orientation angles $\varphi_{W}$ and $\varphi_{R}$ of the beam spot ellipse have been validated by the commercial beam optics Code V and are found to be in good agreement.
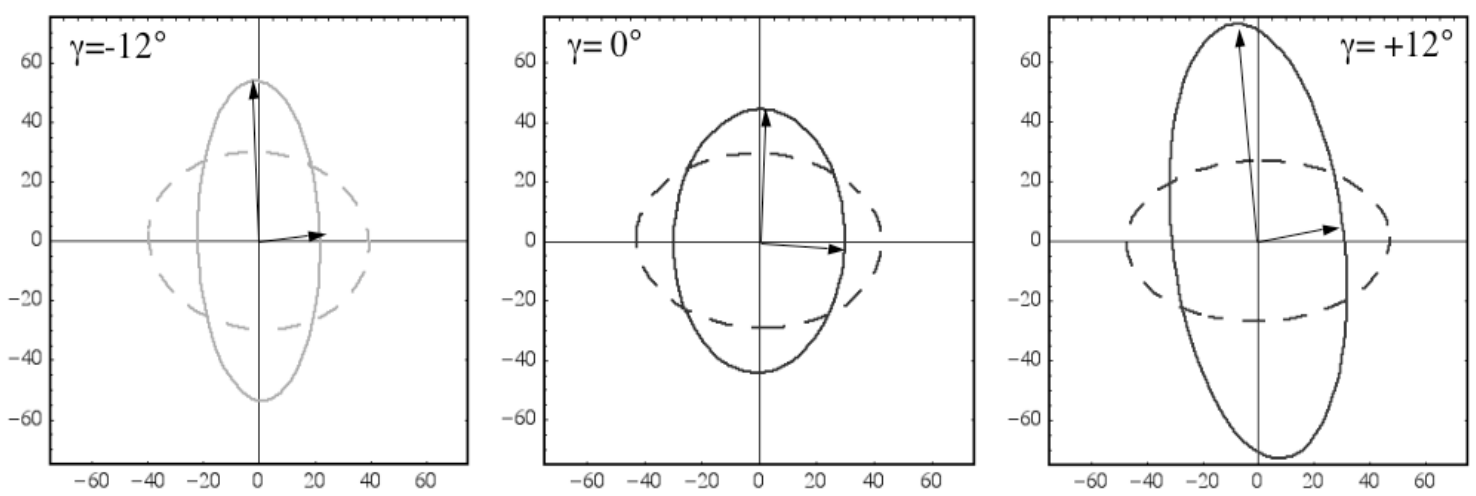

Figure 3. A cross sectional view of the beam spot orientation (upper row of beams) is shown, just after reflection at mirror M2 (dashed line) and at the absorption region (solid line). The beam spot is shown to turn from flat to near upright position at the absorption region. From left to right three cases of beam steering $\gamma$ are shown: $\varphi_{W}\left[\gamma=-12^{\circ}\right]=+1.4^{\circ}, \varphi_{W}\left[\gamma=0^{\circ}\right]=-1.5^{\circ}$ and $\varphi_{W}\left[\gamma=12^{\circ}\right]=+7.1^{\circ}$.

\subsection{Beam tracing by the TORBEAM code}

In this section, the propagation of the mm-waves through the plasma is described. The parameters necessary to define the initial condition of the TORBEAM calculations are given by the poloidal and toroidal injection angles, the coordinates of the reflection points on mirror M2, the principal beam widths in the two astigmatism directions, the principal curvature radii of the reflecting wave fronts and the orientation angles $\varphi_{W}$ and $\varphi_{R}$ just after reflection on mirror M2. In the TORBEAM code, a central ray is advanced through the cold plasma dispersion relation by calculating the Jacobian. A complex eikonal describes the finite beam size allowing for a (weak) spread in the refractive index over the beam cross section. Absorption of the beam is calculated along the central ray in the weakly relativistic approximation [27]. Current drive follows from an analytic solution to the adjoint equation [28]. The equilibrium bootstrap current is derived from simulations of the plasma scenarios considered, employing the ASTRA code version 5.2. 
Plasma diffraction effects are taken into account in the paraxial WKB (pWKB) approximation, through a complex wave phase (eikonal), in which the imaginary part describes of the transverse structure of the beam. The electric field of the beam is written in the form

$$
\vec{E}(\vec{r})=A(\vec{r}) \vec{e}(\vec{r}) \exp [i(s(\vec{r})+i \phi(\vec{r}))],
$$

where $A$ is the amplitude, $\vec{e}$ the unit polarization vector and $s+i \phi$ is the complex eikonal. A paraxial expansion of the eikonal around the beam maximum (which obeys the equations of geometric optics and is therefore called the central ray), is justified if the properties of the medium do not change significantly across the beam cross section. This allows the phase to be written as:

$$
\begin{aligned}
& s(\vec{r})=s_{0}(\vec{r})+K_{\alpha}\left[x_{\alpha}-q_{\alpha}(\vec{r})\right]+\frac{1}{2} s_{\alpha \beta}\left[x_{\alpha}-q_{\alpha}(\vec{r})\right]\left[x_{\beta}-q_{\beta}(\vec{r})\right] \\
& \phi(\vec{r})=\frac{1}{2} \phi_{\alpha \beta}\left[x_{\alpha}-q_{\alpha}(\vec{r})\right]\left[x_{\beta}-q_{\beta}(\vec{r})\right],
\end{aligned}
$$

where $q_{\alpha}$ and $K_{\alpha}$ are the components of the position and of the wave vector calculated on the reference ray respectively (sum over repeated indices is assumed). The second-order quantities $s_{\alpha \beta}$ and $\phi_{\alpha \beta}$ are connected to the curvature of the beam front and to the width of the beam cross section. In particular, the quadratic form defining $\phi$ is positive definite. Its contour levels are ellipses, with the principal axes representing the principal beam widths.

Generally, the matrices $s_{\alpha \beta}$ and $\phi_{\alpha \beta}$ are not diagonal. However, it can be shown that in a (local) reference frame in which the beam propagates along the $x$ axis, the elements of the first row and the first column $\phi_{x \alpha}(\alpha=1,2,3)$ must vanish. In this coordinate frame the angle $\theta$, defined as $\tan (2 \theta)=2 \phi_{y z} /\left(\phi_{y y}-\phi_{z z}\right)$, can be interpreted as the rotation angle of the principal axes of the beam cross section with respect to a horizontal plane. A similar rotation can be introduced for the phase front using the elements $s_{\alpha \beta}$. In general, the resulting angle will not coincide with the angle $\theta$ defined above, a characteristic of general astigmatism, since (a) it has no cylindrical symmetry (astigmatism) and (b) the focusing directions are not aligned with the principal axes of the beam cross section. A beam tracing approach based on $\mathrm{pWKB}$ expansion allows a very elegant description of generally astigmatic beams, since it is based on the knowledge of the matrix elements $s_{\alpha \beta}$ and $\phi_{\alpha \beta}$, which are directly obtained as the solution of the beam tracing equations [22].

In a plasma, beams become generally astigmatic as a consequence of the anisotropic dispersion properties of the plasma [29]. However, under typical ITER ECCD conditions, where the wave frequency is significantly above the plasma frequency, the rotation angle $\theta$ is mainly determined by the launch conditions whilst the influence of the plasma turns out to be fairly small [30]. This interesting result would permit neglect of the plasma effect in future $\eta_{n t m}$ performance assessment.

The combined general astigmatic calculation of beam propagation inside the launcher and through the plasma results in an elongated beam profile, see Figure 3. As pointed out before, this shape of the beam spot ellipse elongated in vertical (poloidal) direction at the absorption region has an unfavourable effect on the profile of current drive which requires a small beam dimension in vertical direction. It should be noted that the effect of a broad deposition profile (comparable to or in excess of the island width) is taken into account in the $\eta_{n t m}$ performance calculation and does not change the $\eta_{n t m}$ $>1.2$ stability criterion [9]. The TORBEAM results have been compared with the GRAY code [26] and are found to agree within 10\%, the GRAY code results being on the favourable side for NTM stabilisation performance. The GRAY code takes into account strong non-uniformities across the beam cross section by calculating plasma absorption independently along each ray of the quasi-optical propagation model. 
Future designs may exploit beam rotation of the launcher by reducing the poloidal beam dimension at the resonant q surface. This requires an increased beam rotation prior to the front mirror M2. This maybe accomplished by the steering mirror system.

\section{Results of improvements to the RS design}

In this chapter, options to improve the performance of the Remote Steering concept are presented. The limitation in $\eta_{n t m}$ performance is determined primarily by the square corrugated waveguide SCW where beam focusing deteriorates at extreme steering angles. Enhanced performance can be achieved in a number of ways:

- Increasing the SCW dimensions

- Optimization of mirror shapes

- Optimization the SCW shape by employing tapers

- Dual launcher concept

- Optimization UPL steering range for access to the $\mathrm{q}=1$ flux surface

- Omitting the front mirror assembly by bending the SCW

Including general astigmatism in the calculation of beam propagation is necessary for the validation of the performance calculations. From the previous section it is clear that general astigmatism has a relatively modest effect on the $\eta_{n t m}$ performance values calculated. Therefore, in the optimizations carried out in sections 4.2 and 4.3 general astigmatism has been neglected. In section 4.1 the general astigmatism has been included for comparison with results obtained earlier [15] where general astigmatism had not been taken into account. Section 4.4, the final results Table 4 again includes general astigmatism.

\subsection{Increasing the SCW dimensions}

Designs presented earlier include a short and a long SCW variant [15]. The long waveguide variant allows for a wider waveguide cross section that improves the beam quality at extreme steering angles. As predicted, by doubling the length of the SCW, the spot-size (averaged over the steering range $z$ ) improves by a factor of $\sim \sqrt{ } 2$ at equal steering range.

Results of the $\eta_{n t m}$ performance calculation for the long and short SCW design options in the ITER reference scenarios are presented in Table 1. Calculations include general astigmatism for the first time. The calculation is carried out for mechanically and thermally consistent designs; for the long SCW variant with steering in vertical direction ranging from $z=1900 \mathrm{~mm}$ to $3300 \mathrm{~mm}$ and for the short SCW variant with steering limited from $z=2400 \mathrm{~mm}$ to $3300 \mathrm{~mm}$. The steering range in the short SCW variant was reduced in favour of better $\eta_{n t m}$ performance in the remaining scenarios. The relaxation in NTM stabilisation requirements is realised by abandoning the requirement for NTM stabilisation at the $q=3 / 2$ surface of scenario $3 \mathrm{a}$. This relaxation of requirements may be justified by the relative small impact it has on ITER performance.

Table 1 presents the peak ECCD current density for each row of beams, scenario and rational surface of interest (located at a normalized poloidal radius $\rho_{p}$ ), assuming that $10 \mathrm{MW}$ is injected from the upper row and $10 \mathrm{MW}$ from the lower row of beams. In the last column, the number in brackets includes a correction factor (ranging between $4 \%$ and $12 \%$ for the scenarios considered here) which accounts for different definitions of the current density in the beam tracing codes (wave driven current) and in the ASTRA code version 5.2 calculating the bootstrap current. This correction has a positive effect on $\eta_{n t m}$ performance. The figure without brackets corresponds to $\eta_{n t m}$ values in Tables 2 to 4 which do not include the current density correction factor (and are therefore pessimistic).

Table 1. TORBEAM results for the "short-waveguide" (limited range) launcher A1 and the "double-length waveguide" A2. Current densities are expressed in $\mathrm{MA} / \mathrm{m}^{2}$. The normalised plasma radius $\rho_{\mathrm{p}}$ is defined as the square root of the poloidal flux surface. The toroidal injection angles vary for the long SCW from 
approximately $17.3^{\circ}$ to $20.2^{\circ}$ and for the short $\mathrm{SCW}$ from $15.7^{\circ}$ to $20.1^{\circ}$ the lower injection angles pointing to deeper lying $\mathrm{q}=3 / 2$ surfaces.

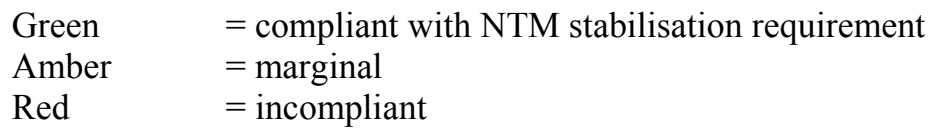

A1 Short length Square Corrugated Waveguide, limited z-range, spherical mirrors

A2 Double length Square Corrugated Waveguide, full z-range, spherical mirrors

\begin{tabular}{|c|c|c|c|c|c|c|c|c|}
\hline Scenario, $q$ & $\rho_{p}$ & $\sim z$ & Row & $j_{b s}$ & $\begin{array}{l}\mathbf{A 1} \\
j_{c d}\end{array}$ & $\begin{array}{c}\mathbf{A 1} \\
\eta_{N T M}\end{array}$ & $\begin{array}{l}\text { A2 } \\
j_{c d}\end{array}$ & $\begin{array}{c}\text { A2 } \\
\eta_{N T M}\end{array}$ \\
\hline \multirow[t]{3}{*}{ EOB5, $q=2 / 1$} & 0.899 & 3342 & Upper & & 0.054 & & 0.053 & \\
\hline & & & Lower & & 0.053 & & 0.057 & \\
\hline & & & & 0.096 & 0.107 & $1.11(1.24)$ & 0.110 & $1.15 \quad(1.28)$ \\
\hline \multirow[t]{3}{*}{$\mathrm{EOB} 2, q=2 / 1$} & 0.872 & 3104 & Upper & & 0.073 & & 0.069 & \\
\hline & & & Lower & & 0.065 & & 0.068 & \\
\hline & & & & 0.073 & 0.139 & $1.90 \quad(2.08)$ & 0.137 & $1.88 \quad(2.05)$ \\
\hline \multirow[t]{3}{*}{ EOB3a, $q=2 / 1$} & 0.836 & 2746 & Upper & & 0.067 & & 0.068 & \\
\hline & & & Lower & & 0.057 & & 0.070 & \\
\hline & & & & 0.119 & 0.123 & $1.03 \quad(1.11)$ & 0.138 & $1.16 \quad(1.24)$ \\
\hline \multirow[t]{3}{*}{ EOB5, $q=3 / 2$} & 0.810 & 2955 & Upper & & 0.046 & & 0.046 & \\
\hline & & & Lower & & 0.039 & & 0.047 & \\
\hline & & & & 0.112 & 0.084 & $0.75 \quad(0.81)$ & 0.093 & $0.83 \quad(0.90)$ \\
\hline \multirow[t]{3}{*}{$\mathrm{EOB} 2, q=3 / 2$} & 0.764 & 2537 & Upper & & 0.034 & & 0.039 & \\
\hline & & & Lower & & 0.030 & & 0.045 & \\
\hline & & & & 0.092 & 0.064 & $0.70 \quad(0.74)$ & 0.084 & $0.91 \quad(0.97)$ \\
\hline \multirow[t]{3}{*}{ EOB3a, $q=3 / 2$} & 0.734 & 2179 & Upper & & - & & 0.041 & \\
\hline & & & Lower & & - & & 0.047 & \\
\hline & & & & 0.145 & - & & 0.088 & $0.61 \quad(0.63)$ \\
\hline
\end{tabular}

On the $q=2 / 1$ surface, the performance calculated is close to or above the requirement $\eta_{n t m}>1.2$. For the $q=3 / 2$ surface $\eta_{n t m}$ values do not meet the criterion for complete NTM stabilization. This is because the beam has broadened when it reaches the absorption region at this more inward lying flux surface. In addition, the value of the bootstrap current is higher toward the centre of the plasma. In the case of the double length SCW, the $q=3 / 2$ surface of scenario $3 \mathrm{a}$ is included in the assessment.

Comparing the two SCW options A1 (short) and A2 (long) reveals that the stabilization efficiency $\eta_{n t m}$ calculated for $q=2 / 1$ surface for scenario $3 \mathrm{a}$, the long waveguide system has now become compliant. Otherwise, the long and short waveguide system do not differ significantly in performance, with values of $\eta_{n t m}$ at the $q=3 / 2$ surfaces still below requirement for the long SCW, albeit improved. These improved values of $\eta_{n t m}$ for the long waveguide at the $q=3 / 2$-surfaces are caused by more tangential toroidal injection angles, i.e. closer to the optimum value of $18^{\circ}$ to $20^{\circ}$ for current drive efficiency (see Table caption).

Further optimization of the long SCW variant is accomplished by the introduction of elliptical mirrors and a zoom mechanism, which yields a reduction in average beam size of $7 \%$ and $14 \%$ respectively [15]. A zoom mechanism decouples the input waist size from the waist position thus producing a beam shape that is optimal for acceptance at the front mirror assembly. Such a steering system is mechanically more complicated because it requires two independently moveable mirrors, rather than one movable mirror in the RS steering mechanism.

Both long and short SCW variants were evaluated on the basis of a number of criteria in addition to performance, including cost, spatial envelope and building constraints, safety aspects of Tritium and Beryllium containment, reliability, maintenance, vacuum and water leak tightness, fatigue and peak 
power loading on the beam line elements [31]. Adding up the various weighing factors, the result of this evaluation shows that the overall advantage of the long over the short SCW variant is only marginal. Because the short SCW variant readily fits within the spatial envelope assigned to the UPL, only the short $\mathrm{SCW}$ variant is pursued.

\subsection{Optimisation of mirror shapes}

Improved performance of the short waveguide design can be achieved by employing mirrors with an elliptical cross section in the steering plane. Results of constrained optimization for a limited range RS design $(z=2400-3300 \mathrm{~mm})$ with constant curvature mirrors (option B) and with non-constant curvature mirrors (option B1) are shown in Table 2 and Figure 4. A 5\% reduction in average beam size over the $z$-range is achieved yielding a $4 \% \eta_{n t m}$ gain. This performance gain is similar in magnitude to the $7 \%$ gain reached for the long SCW option. An additional zoom option in the steering system would yield a further improvement of $\sim 14 \%$.

As is evident from Table 2, the performance of the $q=3 / 2$ scenarios is found to be below the marginal value $\eta_{n t m}=1$. The performance of scenario $5 q=3 / 2$ could be brought closer to the desired level by a further reduction in the steering range to $z=2600-3300$. For this "tightened limited range" option B2, the average beam size reduction is $25 \%$ resulting in an $11 \% \eta_{n t m}$ gain for the $\mathrm{q}=1$ scenarios, see Section 4.5. The improvement for the $\mathrm{q}=3 / 2$ at scenario 5 is only marginal. In this option there is no access to the $q=3 / 2$ surface of scenario 2 .

The maximum heat load on the front mirror $\mathrm{M} 2$ is reduced from 5.7 to $5.3 \mathrm{MW} / \mathrm{m}^{2}$ for option $\mathrm{B} 1$ and to $4.7 \mathrm{MW} / \mathrm{m}^{2}$ for option B2. Further optimization in performance by reducing the performance at the $q=2$ scenarios at the expense of the $q=3 / 2$ scenarios, has the potential of reaching $\eta_{n t m}>1.2$ for the remaining $q=3 / 2$ scenario 5 .

Table 2. TORBEAM $\eta_{n t m}$ performance results for the selected cases B, B1 and B2.

In all cases, the configuration of the steering system is unchanged.

B limited range optimized $\mathrm{z}=2400-3300 \mathrm{~mm}$, spherical mirrors

B1 re-optimization on B including mirrors with elliptical cross-section

B2 re-optimization on B1 with a "tightened" limited range ( $\mathrm{z}=2600-3300 \mathrm{~mm})$

\begin{tabular}{|l|c|c|c|c|c|}
\hline \multicolumn{1}{|c|}{ Scenario } & $q$ & $\sim z$ & B & B1 & B2 \\
\hline EOB5 & $2 / 1$ & 3342 & 1.22 & 1.31 & 1.45 \\
\hline EOB2 & $2 / 1$ & 3104 & 1.74 & 1.89 & 2.03 \\
\hline EOB5 & $3 / 2$ & 2955 & 0.84 & 0.87 & 0.88 \\
\hline EOB3a & $2 / 1$ & 2746 & 1.17 & 1.18 & 1.16 \\
\hline EOB2 & $3 / 2$ & 2537 & 0.93 & 0.88 & N.A. \\
\hline $\begin{array}{l}\text { Max heat load in z-range } \\
\text { on M1, M2 [MW/m }{ }^{2} \text { ] }\end{array}$ & & & $4.6,5.7$ & $3.6,5.3$ & $3.0,4.7$ \\
\hline $\begin{array}{l}\text { Average beam size on } \\
\text { resonance layer UR, LR [mm] }\end{array}$ & & & $77.3,90.1$ & $74.4,85.2$ & $58.4,66.3$ \\
\hline
\end{tabular}




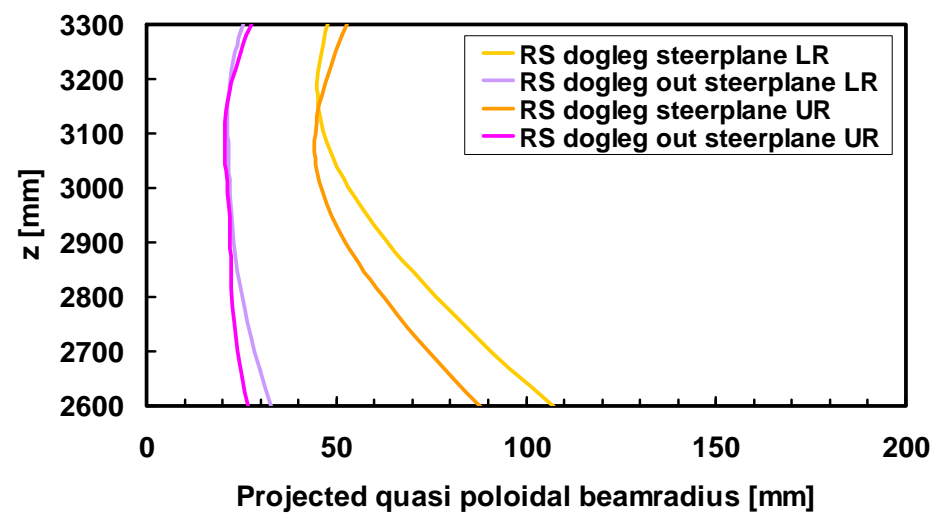

Figure 4. Projection of the beam radius on the resonance plane as a function of the ITER $z$ coordinate for the scenarios indicated in Table 2. Result of the "tightened limited range" ( $z=2600-3300 \mathrm{~mm})$ launcher B2 is shown. Vacuum propagation is assumed. Propagation of the Upper Row (UR) and Lower Row (LR) of beams is shown. Beam dimensions in the steering (poloidal) plane (right) and in the plane perpendicular to the steering plane (left) are shown.

\subsection{Optimization the SCW shape by employing tapers}

The output steering range of an SCW may be enhanced (or reduced) by using a down- (or up-) tapering of the waveguides in the steering plane [32]. However, an enhanced beam steering range goes at the expense of beam focussing, in this case because the input/output ratio of the waist of the beam scales with the input/output dimensions of the tapered waveguide, yielding a smaller output beam waist at larger steering range. A smaller output beam waist results in a higher beam divergence and consequently a wider beam deposition profile at the resonance plane. These conflicting effects in terms of performance need to be optimised. Optimizations were carried out, with an up- and down taper of the length of a short SCW.

Option T1 considers a down-taper with an input and output dimension of 48.4 and $40 \mathrm{~mm}$ respectively. Option T2 considers an up-taper with an input and output dimension of 40 and $48.4 \mathrm{~mm}$. In both options the optics has been optimised. Finally, in option T3 the taper angle was included as a free (but constrained) parameter in the optimisation, together with the optics. The output aperture of the SCW is varied between $34 \mathrm{~mm}$ to $54 \mathrm{~mm}$ symmetrically around the un-tapered SCW aperture of $44 \mathrm{~mm}$ fixed at half length of the SCW [6]. The optimization procedure resulted in different down tapers for the upper and the lower row of beams. The optimized SCW tapers T3 yield an additional reduction in average beam size, but only a marginal gain in $\eta_{n t m}$ performance value.

TORBEAM results of the tapered SCW optimisation in terms of $\eta_{n t m}$ performance are presented in Table 3. As compared with the non-tapered SCW, results of the $q=3 / 2$ surfaces are marginally improved. Interestingly, the optimization of the beam taper option T3 results in slight down tapers of different dimension for the Upper and Lower row of beams.

Table 3. TORBEAM $\eta_{n t m}$ performance results for the selected cases B1 to T3. In all cases the configuration of the steering system is unchanged.

B1 limited range including mirrors with elliptical cross-section (steer plane)

T1 optical optimization B1 using a SCW down taper of $48.4 \mathrm{~mm}$ to $40 \mathrm{~mm}$

T2 optical optimization B1 using an SCW up taper of $40.3 \mathrm{~mm}$ to $48.0 \mathrm{~mm}$

T3 simultaneous optical and taper optimization of B1, resulting in down tapers of 44.9 to $43.1 \mathrm{~mm}$ for the Upper and $46.2 \mathrm{~mm}$ to $41.9 \mathrm{~mm}$ for the Lower beam row respectively 


\begin{tabular}{|l|c|c|c|c|c|c|}
\hline \multicolumn{1}{|c|}{ Scenario } & $q$ & $\sim z$ & B1 & T1 & T2 & T3 \\
\hline EOB5 & $2 / 1$ & 3342 & 1.31 & 1.31 & 1.16 & 1.32 \\
\hline EOB2 & $2 / 1$ & 3104 & 1.89 & 1.88 & 1.68 & 1.86 \\
\hline EOB5 & $3 / 2$ & 2955 & 0.87 & 0.84 & 0.83 & 0.87 \\
\hline EOB3a & $2 / 1$ & 2746 & 1.18 & 1.12 & 1.19 & 1.21 \\
\hline EOB2 & $3 / 2$ & 2537 & 0.88 & 0.82 & 0.97 & 0.95 \\
\hline $\begin{array}{l}\text { Max heat load in z-range } \\
\text { on M1, M2 }\left[\mathrm{MW} / \mathrm{m}^{2}\right]\end{array}$ & & & $3.6,5.3$ & $3.6,5.5$ & $4.9,5.5$ & $3.9,5.2$ \\
\hline
\end{tabular}

By introducing a slight modification in the SCW cross-sectional shape the phase slippage of the propagation constants can be compensated for [35]. As a result, the total steering range of the SCW can be increased from $+/-12^{\circ}$ to approximately $+/-18^{\circ}$. This design option would require an enlarged steering range of the remote steering system and a reduction in distance from SCW exit to the first front mirror M1 in order to keep the beam footprint within the mirror boundaries over the increased steering range, where the size of mirror M1 is constrained by the spatial envelope of the port plug. This design option would result in a higher heat load on the front mirrors and has not been studied in detail.

\subsection{Dual launcher concept}

Two sets of launchers optimised for reaching the $q=2$ and $q=3 / 2$ surfaces separately provide flexibility in the optimization resulting in higher performance, provided there is freedom in the distribution of the power over the launchers. Recently, this approach has gained interest by the prospect of $2 \mathrm{MW}$ gyrotrons becoming available in the near future, either by applying power combiners [36] or by doubling the gyrotron power. A "complementary range" launcher B3, complementary to the "limited range" launcher B forms the basis of this RS concept. In this launcher concept the scenarios 3a and 2 at $q=3 / 2$ are constrained parameters in the optics optimisation, which is optimized to reach the lowest $z$ $=1600 \mathrm{~mm}$ value. In this way the $q=1$ surface can potentially be reached, but would require a modification in the mechanical design of the ITER adjacent lower blanket section, which would need an enlarged beam entrance aperture for the beams to be steered to the $q=1$ surface.

Results of this dual launcher system B3 with complementary ranges are presented in Table 4 and Figure 5. As can be seen from the table, the deep lying $\mathrm{q}=3 / 2$ surfaces can be made fully compliant. Compared with Table 2 the option B numerical values differ in that in Table 4 general astigmatism is included in the performance calculation.

Table 4. TORBEAM $\eta_{n t m}$ performance results (general astigmatic calculation) for the selected cases B and B3. In all cases, the configuration of the steering system is unchanged and spherical mirrors are employed.

B "limited range" option with $z=2400-3300 \mathrm{~mm}$

B3 “complementary to B" option with $z=\sim 1600-2600 \mathrm{~mm}$

\begin{tabular}{|l|c|c|c|c|}
\hline \multicolumn{1}{|c|}{ Scenario } & $q$ & $\sim z$ & B (gen astigm) & B3 \\
\hline EOB5 & $2 / 1$ & 3342 & 1.24 & \\
\hline EOB2 & $2 / 1$ & 3104 & 2.08 & \\
\hline EOB5 & $3 / 2$ & 2955 & 0.81 & \\
\hline EOB3a & $2 / 1$ & 2746 & 1.11 & \\
\hline EOB2 & $3 / 2$ & 2537 & 0.74 & 2.22 \\
\hline EOB3a & $3 / 2$ & 2179 & & 1.46 \\
\hline $\begin{array}{l}\text { Max heat load in z-range } \\
\left.\text { on M1, M2 [MW/m }{ }^{2}\right]\end{array}$ & & & $4.6,5.7$ & $2.8,5.1$ \\
\hline
\end{tabular}




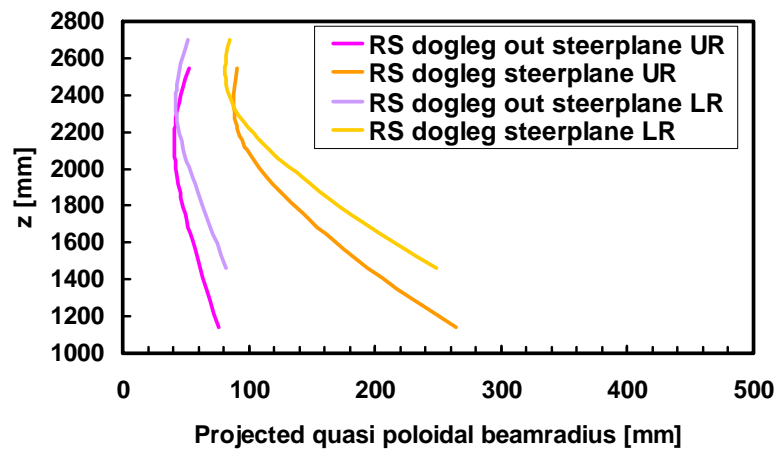

Figure 5. Projection of the beam radius on the resonance plane as a function of the ITER $\mathrm{z}$ coordinate for the scenarios indicated in Table 4 for the complimentary version of the "tightened limited range" optimized launcher ( $\mathrm{z}=\sim 1600-2600 \mathrm{~mm}$, option B3). The Upper Row (UR) and Lower Row (LR) results are shown. Beam dimensions in the steering (poloidal) plane (right) and in the plane perpendicular to the steering plane (left) are shown. Vacuum propagation is assumed.

\subsection{Optimization UPL steering range for access to the $q=1$ flux surface}

Sawtooth control has become an increasingly important issue for the ECCD system on ITER. Experiments have shown that sawtooth control can prevent a sawtooth crash from triggering an NTM. Therefore, in order to preempt the growth of NTMs, it would be advantageous to add sawtooth control to the ITER UPL ECCD system requirements.

The ITER Project Integration Document does not explicitly demand control of the sawtooth by either the EC Upper or Equatorial launcher, but implicitly does by defining the access ranges as $0<\rho_{p}<0.5$ for the Equatorial Launcher (EL) and $0.4<\rho_{p}<0.9$ for the Upper Launcher (UL). A future upgrade of the ECCD system to an installed power of $48 \mathrm{MW}$ would allow simultaneous operation of the EL for bulk current drive and the UPL for NTM stabilization and sawtooth control.

The sawtooth period is affected by driving current just in or outside the $q=1$ rational surface. This additional localized non-inductively driven current changes the value of the shear at the location of the $q=1$ surface and thus influences the sawtooth period. A criterion by Merkulov [10] has been defined such that the sawtooth period is significantly affected by the driven current when the condition $I_{c d}>$ $1 / 2\left(\mathrm{w}_{\mathrm{cd}} / \rho_{q=1}\right)^{2} I_{q=1}$ is satisfied. This criterion does not include the stabilizing effect of alpha particles and therefore is quantitatively incorrect. However, the criterion is useful for a qualitative comparison of different launcher design options. The expected location of the $q=1$ surface varies between $0.4<$ $\rho_{p}<0.45$ for scenario 2 and between $0.35<\rho_{p}<0.4$ for scenario $3 \mathrm{a}$.

According to [10] the shear around $q=1$ can be most profitably manipulated by ECCD generated at a value of $\rho_{l}$ which is $25 \%$ higher than $\rho_{q=1}$ As shown in Table 5, the most inward lying flux surface that can be reached by both upper and lower row of beams is located at $\rho_{l}=0.5$. Therefore, the shear at the $\mathrm{q}=1$ surface lying at $\rho_{q=1}=0.4$ can be lowered by co-drive ECCD and raised by counter-drive ECCD directed at the $\rho_{l}=0.5$ flux surface. Table 5 has been evaluated assuming that the $q=1$ surface lies on the flux surface $\rho_{p}=\rho_{l}$. The driven current for the upper and lower row is summed and the deposition width $w_{c d}$ is taken as an average of the two rows of launchers as derived by TORBEAM calculations.

Table 5. Evaluation of launcher B3 for sawtooth control

\begin{tabular}{|l|l|l|l|l|l|}
\hline \multicolumn{1}{|c|}{ Scenario } & \multicolumn{1}{c|}{$\rho_{l}$} & \multicolumn{1}{c|}{$I_{c d}[\mathrm{MA}]$} & \multicolumn{1}{c|}{$w_{c d}$} & \multicolumn{1}{c|}{$I_{q=1}[\mathrm{MA}]$} & $1 / 2\left(w_{c d} / \rho_{q=1}\right)^{2} I_{q=1}$ \\
\hline 2 & 0.50 & 0.121 & 0.087 & 9.5 & 0.144 \\
\hline $3 \mathrm{a}$ & 0.52 & 0.212 & 0.065 & 7.5 & 0.063 \\
\hline
\end{tabular}


The Merkulov criterion is fulfilled for scenario $3 \mathrm{a}$, and is almost fulfilled for scenario 2 . The cause lies with the lower row of beams, unable to reach the $q=1$ surface in scenarios 2 and 3a. However, by reducing the large margin that presently exists in the NTM-stabilization performance for scenario 2 (see Table 4), the range of the sawtooth launcher may be redirected to include the more inward lying $\rho=0.35$ flux surface and so fulfill the Merkulov criterion for scenario 2 .

\subsection{Omitting the front mirror assembly by bending the SCW}

A critical element in the RS design concept is the front mirror assembly, both because the power loading is relatively high and because the blanket aperture is relatively narrow setting spatial constraints to the mirror size and thus power loading. Recently, it has been suggested [33] that the front mirror assembly may be disposed of all together by bending the SCW, such as to acquire the desired access angle to the plasma without the need for a front mirror assembly, whilst maintaining neutron shielding requirements.

Preliminary simulations were carried out by using a SCW bending angle of about $18^{\circ}$, employing the IAP waveguide synthesis code [18]. The dimensions of the SCW are taken $50 \mathrm{~mm}$ wide and a total length of $5590 \mathrm{~mm}$. The result of SCW transmission efficiency as a function of launcher steering angle is shown in Figure 6. Unfortunately, these results show that the SCW mode field structure becomes corrupted. The mode structure deteriorates with increased bending angle. In addition, the propagation constants are shifted far from their optimal straight waveguide values. These two effects reduce the transmission efficiency as a function of launching angle to low values. Although efficient bending would be possible in some applications, a SCW bend of this form is not suitable for ITER where a variable launcher steering angle is essential for NTM control.

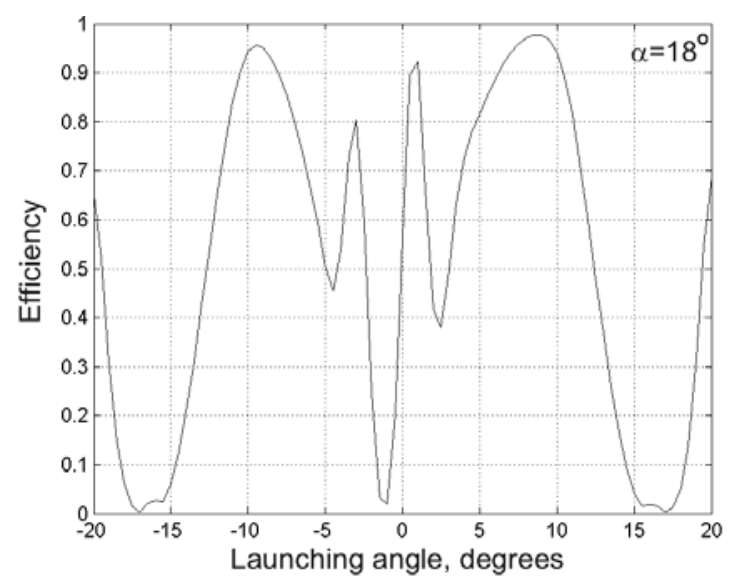

Figure 6: SCW transmission efficiency of an initial (sinusoidal) wave field versus launching angle. The SCW is bent over an angle of $18^{\circ}$, which can be oriented such as to suit the required toroidal and poloidal launch direction for ITER. SCW length $\mathrm{L}=5590 \mathrm{~mm}$ and width $=50 \mathrm{~mm}$.

\section{Discussion and Future developments}

\subsection{Validity of computations}

The question arises as to what the accuracy is of the $\eta_{n t m}$ values calculated, in view of the magnitude of performance enhancements that are being discussed in the optimisation of the RS launcher. This question has been addressed by a benchmark study comparing 11 computer codes, calculating the electron cyclotron current drive under ITER conditions [37]. The codes differ in their mm-wave propagation scheme (ray tracing, Gaussian or quasi-optical), the plasma absorption (analytical and 
Fokker-Planck in quasi-linear approximation) and current drive (analytic adjoint method or FokkerPlanck first moment). Relativistic effects, Doppler shifting the electron cyclotron resonance, are included in a weak or full relativistic manner. The study includes the TORBEAM code employing the weakly relativistic absorption model, used throughout this paper [27].

The electron cyclotron wave driven current density profile has been calculated by the codes reviewed. It transpires that the peak current density varies by approximately $+/-25 \%$ over the range of codes and that the profile width varies by approximately $+/-15 \%$. Total integrated current shows a spread of approximately $+/-5 \%$. The current density profile is the most sensitive parameter in the $\eta_{n t m}$ calculation, as current drive needs to be confined to the island width around the rational $q$ surface.

Validation of the codes by experiment is carried out on DIII-D [38] and on JT-60U [39] . The driven current is derived from Motional Stark Effect polarimetry, which measures the change in magnetic field pitch angle to derive the toroidal current density. The conditions under which the validation is carried out for DIII-D differ a factor 3 to 4 in electron density and temperature compared with ITER, whilst on JT-60U the electron temperature is comparable with ITER values, significant for EC wave absorption. For DIII-D the results of co-ECCD agree well with theory, whilst for counter injection there is $10 \%$ lower current drive than predicted. For JT-60U, the measured current drive is $40 \%$ down on calculation. This is attributed to the transient nature of the discharge. Current drive efficiency requires a factor 5 extrapolation from JT-60U to reach ITER values.

Obviously, the discrepancy between codes is of a systematic nature. Precision (relative error) of the codes is expected to be in the percentage range. Therefore, optimisation of performance in the $10 \%$ range, as carried out in this paper, will be meaningful. The validation of ECCD by experiment requires a modest but significant extrapolation to ITER conditions. It is important to note that the relevant parameter for ECCD stabilization of NTMs refers to the current density profile whilst experimental validation refers to the total current driven. Furthermore, the bootstrap current necessary for calculating $\eta_{n t m}$ adds to the error bar. To allow for this uncertainty the $\eta_{n t m}=1.2$ criterion includes a $20 \%$ safety margin, which is expected to cover the uncertainty range.

\subsection{Future developments}

A few options for performance enhancement of the RS concept remain open, as reviewed in this article, including:

- A minor modification to the cross-sectional shape of the SCW will allow for a larger steering range by increasing the steering angle into the SCW from 12 degrees to 18 degrees.

- Including a zoom mechanism improves the $\eta_{n t m}$ performance but requires a more complicated steering unit.

- Removing part of the vertical side walls of the SCW would produce a larger beam divergence perpendicular to the steering plane in favour of a reduced heat load on the front mirrors.

- An optimised $\eta_{n t m}$ trade off over the (limited) steering contains room for improvement of marginal $\eta_{n t m}$ values at some scenarios at the expense of high $\eta_{n t m}$ values at other scenarios.

- The concept of dedicated launchers may be further explored with the anticipated increase in gyrotron power and the fast switching of mm-wave power between launchers becoming available in the near future.

Current launcher designs (and certain diagnostics) are limited in performance by the access geometry allocated to the ITER Upper Port Plugs. It can be shown that lowering the access port of the ITER Upper Port Launcher to the adjacent lower blanket section, would significantly improve the range of q surfaces accessible by the mm-wave beams and thus enhance performance [34]. This potential improvement of the ECCD system would benefit the RS design concept to a larger degree compared with FS. However, such a change in blanket design has severe impact on the overall ITER design. It has not been adopted at the 2007 ITER baseline review. 
A crucial element in the RS concept is the SCW, which allows for a compact design solution but limits the performance of the launcher by the steering range available. This limitation could be overcome by a design in which the SCWs are replaced by a set of mirrors in a (near) confocal arrangement (including the front mirrors and steering mirrors) to form a multi-beam quasi-optical transmission line with no waveguides [18]. In such an arrangement, the waist magnification equals the ratio of the focal distances of the mirror system. A special feature of the confocal mirror arrangement is that good focussing properties can be achieved over an extended range of beam steering. A preliminary design has been produced. The port plug integration and neutron shielding requires further design work to be carried out.

Discussion so far has concentrated on NTM stabilisation by continuous mm-wave power radiated on the location of the instability. Modulation of the mm-wave power, synchronous with the rotation of the magnetic island, may enhance the effectiveness of NTM stabilization [9]. NTM stabilization by modulated mm-wave power will be explored in a separate paper.

\section{Summary and Conclusions}

The NTM stabilisation performance predicted for Remote Steering based ECCD on ITER is presented in Table 4. The dual launcher concept dedicated to individual $\mathrm{q}=2 / 1$ and $\mathrm{q}=3 / 2$ surfaces exceeds the requirement $\eta_{n t m}>1.2$ for full NTM stabilisation in four out of the six cases considered with one scenario being marginally compliant and one scenario non-compliant. The effectiveness of the RS launcher for sawtooth control is marginal but there is scope for improvement by a better trade off in $\eta_{n t m}$ values. Although all options considered are compatible with the ITER Upper Port Plug, the complementary low $\mathrm{z}$ launchers would need an additional modification of the adjacent lower blanket section. All power loading calculations presented in this paper are based on $2 \mathrm{MW}$ rated gyrotrons per beam line, i.e. anticipating on a future power upgrade to $48 \mathrm{MW}$ EC beam power installed at ITER.

The proposed design solution based on Remote Steering is a fallback option in case the ITER baseline design based on Front Steering meets with an unforeseen showstopper.

Potential improvements to the RS design such as the application of non-spherical mirror curvatures, tapered SCWs, curved SCWs and the use of general astigmatic beams have been investigated. The findings reported in this paper can be summarized as follows:

- The RS option with non-spherical front mirrors yields an improvement of about $5 \%$ in $\eta_{n t m}$ performance above similar designs without this refinement;

- Additional application of a tapered SCW adds another 3\% to the $\eta_{n t m}$ performance;

- Inclusion of general astigmatism in beam transport calculations modifies the predicted performance by typically $10 \%$;

- The improvements obtained by a longer and wider SCW are marginal when in addition to performance also cost, remote handling and maintenance and building interface requirements are weighed in;

- A poloidally bent SCW omitting the front mirrors in the current launcher design and enhancing plasma accessibility, has been shown not to satisfy the ITER requirements due to its lower and discontinuous transmission efficiency as function of launching angle;

- A "tightened limited range" launcher yields an improvement in $\eta_{n t m}$ performance of $25 \%$ whilst heat load is reduced to $4.7 \mathrm{MW} / \mathrm{m}^{2}$, meeting most of the ITER requirements but at the cost of loosing the capability to stabilize NTMs at the $q=3 / 2$ surface for scenario 2 with the same launcher;

- In case of increased gyrotron power becoming available or with the advent of fast power switching from one launcher to the other separate dedicate launchers become an option: one for $q=2$ NTM stabilization for all scenarios and one for $q=3 / 2$ NTM stabilization with some possibility to control sawtooth at the $q=1$ surface. A cut in the lower shielding blanket is needed to implement this option;

- The combination of limited-range and complementary range launchers exceed the requirement $\eta_{n t m}>1.2$ for full NTM stabilisation in four of the six cases considered and one scenario being marginally compliant. 
If one considers the results of this study on the Remote Steering concept of the ITER Upper Port Launcher and compares these with results of the Front Steering concept, it is obvious that FS outperforms RS, meeting the required amounts of EC-driven current at all relevant q-surfaces. Therefore, it is appropriate to start ITER operation with FS-launchers provided that moving parts can

be constructed that will survive the hostile environment close to the ITER plasma. The later phases of ITER operation are intended as steps towards the development of a DEMO reactor. The FS concept appears at present not feasible for application in DEMO. Therefore, it is suggested to demonstrate the RS-launcher concept during later stages of ITER operations in the light of experience gained on NTM stabilization requirements, especially since at that time it will be known which q-surface NTMs will form the main threat to ITER performance and how much EC-driven current will be actually needed to prevent NTM instability to occur.

\section{Acknowledgements}

The authors are indebted to Prof. F.C. Schüller and the two referees for their helpful comments. This work is supported by the European Communities, under the contract of Association between EURATOM/FOM, and was carried out within the framework of the European Fusion Development Agreement (task TW5-TPHE-ECHULA/B \& TW6-TPHE-ECHULA/B). The views and opinions expressed herein do not necessarily reflect those of the European Commission. 


\section{References}

[1] Henderson M A et al. 2006 The ITER ECH FS Launcher design for an optimized Physics Performance, Proc. 14th Joint Workshop on Electron Cyclotron Emission and Electron Cyclotron Resonance Heating, Santorini, Greece 558-563

[2] Verhoeven A G A et al. 2005 Design of the Remote Steerable ECRH launching system for the ITER upper port J. Phys: Conf. Series 25 84-91

[3] Talbot W H F 1836 Facts Relating to Optical Science No. IV, Phyl. Mag. 9, 56, 401-407.

[4] Moeller C P 1998 A Method of Remotely Steering a Microwave Beam launched from a Highly Overmoded Corrugated Waveguide $23^{\text {rd }}$ International conference on Infrared and Millimeter Waves, Conference Digest, University of Essex, Colchester, UK, 116

[5] Denisov G G, Kuzikov S V, and Kobayashi N 2001 RF Analysis of ITER Remote Steering Antenna for Electron-cyclotron Plasma Heating Int. J. Infrared and Millimeter Waves 2212 $1735-1760$

[6] Bongers W A et al. 2007 Recent Developments of the Upper Port ECH\&CD Launcher Systems for ITER based on the Remote Steering Concept Proc. $4^{\text {th }}$ IAEA Technical Meeting on ECRH Physics and Technology for ITER, Vienna, Austria

[7] Saibene G et al. in Fusion Energy 2006 (Proc. 21st Int. Conf. Chengdu, 2006)

(Vienna: IAEA) CD-ROM file IT/P2-14 and

http://www-aweb.iaea.org/napc/physics/FEC/FEC2006/html/index.htm

[8] Zohm H, Heidinger R, Henderson M, Poli E, Ramponi G, Saibene G and A G A Verhoeven 2005 Comparison of the performance of different options for the ITER ECRH Upper Launcher, J. Phys: Conf. Series 25 234-242

[9] Zohm H et al. 2007 Control of NTMs by ECCD on ASDEX Upgrade in View of ITER Application, Proc. 34th EPS Conference on Plasma Physics, Warsaw, CDROM Published by European Physical Society, Editors: Pawel Gasior and Jerzy Wolowski

[10] Merkulov A, Schüller F C, Westerhof E, de Baar M R, Krämer-Flecken A, Liang Y and TEXTOR Team 2004 Sawtooth period control by localized non-inductive current drive Proceedings of Joint Varenna-Lausanne Int. Workshop on Theory of Fusion Plasmas, Varenna, Italy, J.W. Connor, O. Sauter, and E. Sindoni (Editors), Societa Italiana di Fisica, Bologna, 2004, 279

[11] Serikov A, Fischer U, Heidinger R, Lang K, Luo Y, Tsige-Tamirat H. 2005 Radiation shielding analyses for the ECRH launcher in the ITER Upper Port, $3^{\text {rd }}$ IAEA Technical Meeting on ECRH physics and Technology in ITER J. Phys: Conf. Series 25 181-188

[12] Ronden D M S, van den Berg M A, Bongers W A, Elzendoorn B S Q, Graswinckel M F, Henderson M A, Lamers B, van Nigtevecht K and Verhoeven A G A 2008 Mechanical Design Aspects of a "Long-Waveguide" Version of the Remote Steering ECRH Upper Port Launcher for ITER Fusion Science and Technology 53 104-113

[13] Henderson M, Chavan R, Saibene G, Sauter O, Private Communication, Cumulative full and partial rotation requirements of the Steering Mechanism. EFDA report TW5-TPHEECHULB2/05-1128 dated 1.12.2005

[14] Jonkers B P, Private Communication, UPL-Mirror Thermo-Mechanical Analysis, 2007

[15] Graswinckel M F, van den Berg M A, Bongers W A, Donné A J H, Goede A P H, Lopes Cardozo N, Ronden D M S and Verhoeven A G A 2008 Design of the ITER Upper Port Electron Cyclotron Heating and Current Drive System Based on Remote Steering Fusion Science an Technology 53 208-219

[16] Goldsmith P F 1998, Quasioptical Systems - Gaussian Beams Quasioptical Propagation and Applications, IEEE Press, New York

[17] Chirkov A V, Denisov G G, Kasparek W, Wagner D, Gantenbein G, Haug M and Hollmann F 2001 Simulation and experimental study of a remote steering system for ECRH/ECCD antenna beams Fusion Engineering and Design 53, 465-473

[18] Denisov G G, Kuzikov S et al. 2007, Private communication Summary on possibilities of improvement of RS launchers, IAP Nizhny Novgorod

[19] Bruschi A, Cirant S, Moro A and Sozzi C 2005 Advanced Optics for the Remote Steering ITER ECRH Upper Launcher J. Phys: Conf. Series 25 112-119 
[20] Poli E, Peeters A G and Pereverzev G V 2001 TORBEAM, a beam tracing code for electroncyclotron waves in tokamak plasmas Comp. Phys. Comm. 136 90-104

[21] Pereverzev G V 1996 Reviews of Plasma Physics 19, 1 (Consultants Bureau, New York)

[22] Pereverzev G V 1998 Beam tracing in inhomogeneous anisotropic plasmas Phys. Plasmas 5, 3529-3541

[23] Graswinckel M F and Bongers W A, High and low-power measurements on a remote-steering upper port launcher mock-up Private Communication FOM Institute for Plasma Physics Rijnhuizen

[24] Moro A and Bruschi A 2007 General Astigmatic Beam Propagation in Complex QuasiOptical Launchers: Application to the ITER ECRH Remote Steering Upper Launcher Fusion Science and Technology, 52 No 2 256-265

[25] Arnaud J A and Kogelnik H 1969 Gaussian Light Beams with General Astigmatism Journal of applied optics, 8 No. 8

[26] Farina D 2007 A Quasi-Optical Beam-Tracing Code for Electron Cyclotron Absorption and Current Drive: GRAY Fusion Science and Technology, 52 No. 2 154-160

[27] Westerhof E 1989, Rijnhuizen Report RR-89-183

[28] Cohen R H 1987, Phys. Fluids 30, 2442

[29] Poli E, Pereverzev G V and Peeters A G 1999 Paraxial Gaussian wave beam propagation in an anisotropic inhomogeneous plasma Phys. Plasmas 6, 5

[30] Poli E et al. 2007 Performance Evaluation of the Remote-Steering Option for the ITER EC Upper Launcher, Proc. $4^{\text {th }}$ IAEA Technical Meeting on ECRH Physics and Technology for ITER, Vienna

[31] Ronden D et al. 2007, FOM Institute for Plasma Physics, Private communication, Critical Design Issues, EFDA report 2007

[32] Denisov G G, Kuzikov S V and Kobayashi N 2001, RF Analysis of ITER Remote Steering Antenna for Electron-cyclotron Plasma Heating, Int. J. Infrared and Millimeter Waves 22, 12, $1735-1760$

[33] Henderson M A, Moeller C P 2007 Possible Improvements to a Remote Steering Launcher for Localized Electron Cyclotron Current Drive Fusion Science and Technology 53 220-236

[34] Harvey R W and Perkins F W 2003, Proc. IAEA TM on ECRH Physics \& Technology for ITER, Kloster-Seeon, Germany, CDROM Max-Plank-Institute of Plasmaphysics, Garching Editor H. Zohm

[35] Denisov G G, Fedorova K A, Danilov Yu Yu, Kuzikov S V and Shmelev M Yu 2006 Efficiency Enhancement of Components Based on Talbot Effect, Proceedings of the 31st International Conference on Infrared and Millimeter Waves, Shanghai, China 269, Published by IEEE, Editors Xue Chu Shen et al.

[36] Kasparek W, Petelin M, Shchegolkov D, Erckmann V, Plaum B, Bruschi A and ECRH groups at IPP Greifswald, FZK Karlsruhe and IPF Stuttgart 2007 FaDiS, a Fast Switch and Combiner for High-power Millimetre Wave Beams Proc. $4^{\text {th }}$ IAEA Technical Meeting on ECRH Physics and Technology for ITER, Vienna

[37] Prater R, Farina D, Gribov Yu, Harvey R W, Poli E, Smirnov A P, Volpe F, Westerhof E, Zvonkov A, ITPA Steady State Operation Topical Group 2006, Proc. 14th Joint Workshop on Electron Cyclotron Emission and Electron Cyclotron Resonance Heating, Santorini, Greece, 312

[38] Petty C C, Prater R, Lohr J, Luce T C, Fox W R, Harvey R W, Kinsey J E, Lao L L and Makowski M A 2002 Nucl. Fusion 42, 1366-1375

[39] Suzuki T et al. 2004 Heating and current drive by electron cyclotron waves in JT-60U Nucl. Fusion 44, 699-708 